\title{
Bipartite induced density in triangle-free graphs
}

\author{
Wouter Cames van Batenburg* \\ Department of Computer Science \\ Université Libre de Bruxelles \\ Belgium \\ wcamesva@ulb.ac.be \\ Ross J. Kang ${ }^{\dagger}$ \\ Department of Mathematics \\ Radboud University Nijmegen \\ Netherlands \\ ross. kang@gmail.com
}

\author{
Rémi de Joannis de Verclos ${ }^{\dagger}$ \\ Department of Mathematics \\ Radboud University Nijmegen \\ Netherlands \\ r.deverclos@math.ru.nl \\ François Pirot \\ Department of Mathematics \\ Radboud University Nijmegen \\ Netherlands \\ and
}

LORIA

Université de Lorraine

Nancy, France

francois.pirot@loria.fr

Submitted: Apr 9 2019; Accepted: Dec 19, 2019; Published: May 29, 2020

(C) The authors. Released under the CC BY-ND license (International 4.0).

\begin{abstract}
We prove that any triangle-free graph on $n$ vertices with minimum degree at least $d$ contains a bipartite induced subgraph of minimum degree at least $d^{2} /(2 n)$. This is sharp up to a logarithmic factor in $n$. Relatedly, we show that the fractional chromatic number of any such triangle-free graph is at most the minimum of $n / d$ and $(2+o(1)) \sqrt{n / \log n}$ as $n \rightarrow \infty$. This is sharp up to constant factors. Similarly, we show that the list chromatic number of any such triangle-free graph is at most $O(\min \{\sqrt{n},(n \log n) / d\})$ as $n \rightarrow \infty$.

Relatedly, we also make two conjectures. First, any triangle-free graph on $n$ vertices has fractional chromatic number at most $(\sqrt{2}+o(1)) \sqrt{n / \log n}$ as $n \rightarrow \infty$. Second, any triangle-free graph on $n$ vertices has list chromatic number at most $O(\sqrt{n / \log n})$ as $n \rightarrow \infty$.
\end{abstract}

Mathematics Subject Classifications: 05C35, 05C15

\footnotetext{
* Supported by an ARC grant from the Wallonia-Brussels Federation of Belgium.

†Supported by a Vidi grant (639.032.614) of the Netherlands Organisation for Scientific Research (NWO).
} 


\section{Introduction}

Our starting point is a conjecture of the third author together with Esperet and Thomassé, which would be sharp up to the choice of constant if true. This enticing conjecture was inspired by a list colouring problem.

Conjecture 1.1 (Esperet, Kang, and Thomassé [10]). There is a constant $C>0$ such that any triangle-free graph with minimum degree at least $d$ contains a bipartite induced subgraph of minimum degree at least $C \log d$.

Although the conjecture is new, it might be difficult. Conjecture 1.1 aligns with central challenges in combinatorics, especially about stable sets in triangle-free graphs. For example, iterating a result of Ajtai, Komlós and Szemerédi [1] for triangle-free graphs of given average degree implies Conjecture 1.1 for any triangle-free graph with $O(d)$ maximum degree $^{1}$. The same result (cf. [10, Thm. 3.4]) confirms Conjecture 1.1 for any triangle-free graph on $n$ vertices provided $d=\Omega\left(n^{2 / 3} \sqrt{\log n}\right)$ as $n \rightarrow \infty$.

Our main result is a stronger, near optimal version of this last statement.

Theorem 1.2. There are constants $C_{1}, C_{2}>0$ such that, for $0 \leqslant d \leqslant n / 2$,

- any triangle-free graph on $n \geqslant 2$ vertices with minimum degree at least $d$ contains a bipartite induced subgraph of minimum degree at least $\max \left\{C_{1} d \sqrt{\log n / n}, d^{2} /(2 n)\right\}$; and

- provided $n / d$ is large enough, there is a triangle-free graph on between $n / 2$ and $n$ vertices with minimum degree at least d such that every bipartite induced subgraph has minimum degree at most $\left\lceil C_{2} d^{2} / n\right\rceil \log n$.

Thus we observe the following phase transition behaviour: for $d$ there is a critical exponent of $n$ (namely 1/2) above which we can be assured of bipartite induced minimum degree polynomially large in $n$ and below which we cannot. Theorem 1.2 resolves Problem 4.1 in [10] up to a logarithmic factor. Our constructions for near optimality are blow-ups of an adaptation of Spencer's construction for lower bounds on $R(3, t)$ [23] (see Section 3).

Due to a captivating connection between bipartite induced density and fractional colouring [10], through which high bipartite induced density is guaranteed by small fractional chromatic number (see Theorem 3.1), Theorem 1.2 is closely related to the following extremal result.

\footnotetext{
${ }^{1}$ For the avoidance of any shadow of a doubt for the reader, fix some $0<\varepsilon<1 / 2$ to be specified later, and consider a triangle-free graph on $n$ vertices with minimum degree $d$ and maximum degree $O(d)$. By the result in [1], every induced subgraph on at least $\varepsilon n$ vertices has a stable set of size $x=\lceil C(\varepsilon n \log d) / d\rceil$ for some fixed $C>0$. Thus we can repeatedly extract, so long as at least $\varepsilon n$ vertices remain, disjoint stable sets of size $x$, ultimately yielding a partial proper colouring with at most $(1-\varepsilon) n / x \leqslant(1-\varepsilon) d /(C \varepsilon \log d)$ colour classes, all of size $x$. The number of edges incident to the remaining vertices is $O(\varepsilon n d)$, which can be made smaller than $n d / 4$ for $\varepsilon$ chosen small enough. By the pigeonhole principle, two of the colour classes induce a bipartite subgraph of average degree at least $(n d / 4) /\left(\left(\begin{array}{c}(1-\varepsilon) n / x \\ 2\end{array}\right) \cdot(2 x)\right) \geqslant(C \varepsilon \log d) /\left(4(1-\varepsilon)^{2}\right)$, which in turn contains a bipartite induced subgraph of minimum degree $(C \varepsilon \log d) /\left(8(1-\varepsilon)^{2}\right)$, as desired.
} 
Theorem 1.3. There are constants $C_{1}, C_{2}>0$ such that, for $0<d \leqslant n / 2$,

- any triangle-free graph on $n$ vertices with minimum degree at least d has fractional chromatic number at most $\min \left\{C_{1} \sqrt{n / \log n}, n / d\right\}$;

- provided $n / d$ is large enough, there is a triangle-free graph on between $n / 2$ and $n$ vertices with minimum degree at least $d$ and fractional chromatic number at least $C_{2} \min \{\sqrt{n / \log n}, n / d\}$.

This bound is basic, but has not appeared in the literature as far as we know. There is equality in the $n / d$ bound when $d=n / 2$, in which case we must have a complete bipartite graph with two equal-sized parts. As for the $\Theta(\sqrt{n / \log n})$ bound, it is an interesting problem to sharpen the asymptotic constants. In Section 4, we combine a recent colouring result of Molloy [19] with the proof idea in Theorem 1.3 to show the following as a first step.

Theorem 1.4. As $n \rightarrow \infty$, any triangle-free graph on $n$ vertices has fractional chromatic number at most $(2+o(1)) \sqrt{n / \log n}$.

Note that if one could improve the factor $(2+o(1))$ to $(\sqrt{2}+o(1))$ (which we formally state as a conjecture below), then it would match the best to date asymptotic upper bound for the Ramsey numbers $R(3, t)$ due to Shearer [22]. By the final outcome of the triangle-free process $[3,11]$, Theorem 1.4 is sharp up to a $(2 \sqrt{2}+o(1))$ factor. (The triangle-free process also gives sharpness up to a constant factor in Theorem 1.3.)

Theorem 1.2 is not far from optimal, but the best constructions we know so far are almost regular. As noted above, we already know Conjecture 1.1 in the almost regular case. This motivates the following bound which improves on Theorem 1.2 if the graph is irregular. We prove this in Section 5.

Theorem 1.5. Any triangle-free graph on $n \geqslant 2$ vertices with $m \geqslant 1$ edges and $w_{3}$ (directed) three-edge walks contains a bipartite induced subgraph of minimum degree at least $w_{3} /(4 n m)$.

Observe that $w_{3} \geqslant 2 m d^{2}$ if the graph has minimum degree at least $d$ (with equality for $d$-regular graphs), and so Theorem 1.5 directly implies the $d^{2} /(2 n)$ bound. Put another way, Theorem 1.5 replaces the squared minimum degree term in the bound of Theorem 1.2 by the average over all edges of the product of the two endpoint degrees.

Returning in some sense to the original motivation for this research, our explorations related to Theorem 1.4 have inspired a list colouring analogue of Theorems 1.2 and 1.3, building upon similar ideas.

Theorem 1.6. There is a constant $C>0$ such that any triangle-free graph on $n$ vertices with minimum degree at least $d$ has list chromatic number at most $C \min \{\sqrt{n},(n \log n) / d\}$.

We are unsure of how close this bound is to optimality, but in Section 6 we offer speculation of the correct asymptotic order, in particular for the bound purely in terms of $n$.

Theorems 1.3 and 1.6 inspire the following natural question. 
Problem 1.7. Given a function $d=d(n)$, letting $\chi(n, d)$ denote the largest chromatic number of a triangle-free graph on $n$ vertices with minimum degree at least $d$, asymptotically what is $\chi(n, d) / \min \{\sqrt{n / \log n}, n / d\}$ as $n \rightarrow \infty$ ?

In a sense, substantial effort has already been devoted to this problem when $d$ is linear in $n$, in relation to a problem of Erdős and Simonovits [9], see e.g. [24, 4]. In particular, for $\varepsilon>0$ fixed, the answer is $\omega(1)$ if $d<(1 / 3-\varepsilon) n$ and $d=\Omega(n)$, while it is $O(1)$ if $d>(1 / 3+\varepsilon) n$ or $d=O(\sqrt{n \log n})$. By Theorem 1.6 (or Proposition 6.2 below), the ratio is always $O(\log n)$.

Outline of the paper. This paper is organised as follows. In Section 2 we prove Theorem 1.3, which in turn implies the lower bound of Theorem 1.2 (via Theorem 3.1). In Section 3, we use the Local Lemma to construct a random graph that certifies the near optimality of Theorem 1.2. In the remaining sections we explore generalisations of Theorems 1.2 and 1.3. In Section 4, we prove Theorem 1.4 and we also provide bounds in terms of the number of edges. Section 5 is devoted to the proof of Theorem 1.5 and related results involving the fractional chromatic number of the cube of triangle-free graphs. We discuss and prove Theorem 1.6 in Section 6. Finally, in Section 7 we make some concluding remarks concerning the exclusion of an arbitrary subgraph. In particular, we show how our results generalise to excluding any given cycle as a subgraph.

\section{$1.1 \quad$ Probabilistic preliminaries}

We use some specific forms of the Chernoff bound [13, (2.9) and (2.11)] and the Lovász Local Lemma [23, Thm. 1.3].

Chernoff Bounds. If $0 \leqslant \varepsilon \leqslant 1$, then

$$
\mathbb{P}(|\operatorname{Bin}(n, p)-n p| \geqslant \varepsilon n p)<2 \exp \left(-\varepsilon^{2} n p / 3\right) .
$$

If $x \geqslant 7 n p$, then $\mathbb{P}(\operatorname{Bin}(n, p) \geqslant x)<\exp (-x)$.

$A$ General Local Lemma. Consider a set $\mathcal{E}=\left\{A_{1}, \ldots, A_{n}\right\}$ of (bad) events such that each $A_{i}$ is mutually independent of $\mathcal{E}-\left(\mathcal{D}_{i} \cup A_{i}\right)$, for some $\mathcal{D}_{i} \subseteq \mathcal{E}$. If we have reals $y_{1}, \ldots, y_{n}>0$ such that for each $i$

$$
y_{i} \mathbb{P}\left(A_{i}\right)<1 \quad \text { and } \quad \log y_{i}>\sum_{A_{j} \in \mathcal{D}_{i}} y_{j} \mathbb{P}\left(A_{j}\right),
$$

then the probability that none of the events in $\mathcal{E}$ occur is positive.

\section{Fractional colouring}

Given a graph $G=(V, E)$, we say that a probability distribution $\mathcal{S}$ over the stable sets of $G$ satisfies property $\mathrm{Q}_{r}^{*}$ if $\mathbb{P}(v \in \mathbf{S}) \geqslant r$ for every $v \in V$ and $\mathbf{S}$ taken randomly according to $\mathcal{S}$. Recall that the fractional chromatic number $\chi_{f}(G)$ of $G$ is defined as the smallest $k$ such that there is a probability distribution over the stable sets of $G$ satisfying property $\mathrm{Q}_{1 / k}^{*}$. 
Proof of Theorem 1.3. Let $G=(V, E)$ be a triangle-free graph on $n$ vertices with minimum degree at least $d$. Since the fractional chromatic number is at most the chromatic number, the first term of the upper bound was already observed by Erdős and Hajnal [8] as a consequence of the aforementioned result of Ajtai, Komlós and Szemerédi [1] (see Section 4). For the second term of the upper bound, choose $\mathbf{S}$ from full neighbourhood sets uniformly over all $n$ such sets. Since $G$ is triangle-free, $\mathbf{S}$ is a stable set. For all $v \in V$,

$$
\mathbb{P}(v \in \mathbf{S})=\frac{\operatorname{deg}(v)}{n} \geqslant \frac{d}{n} .
$$

We have shown then that this distribution has property $\mathrm{Q}_{d / n}^{*}$, as required.

For sharpness, fix $\varepsilon>0$ and let $j$ be the smaller of $n$ and the least value for which $2(2+\varepsilon / 4)^{1 / 2} \sqrt{j / \log j} \geqslant n / d$, and consider the final output of the triangle-free process on $j$ vertices. This is a random triangle-free graph that was shown, independently, by Bohman and Keevash [3] and by Fiz Pontiveros, Griffiths and Morris [11], to have minimum degree $\left(\sqrt{2^{-1}}+o(1)\right) \sqrt{j \log j}$ and stability number at most $(\sqrt{2}+o(1)) \sqrt{j \log j}$ with high probability as $j \rightarrow \infty$. For large enough $j$ (which we can guarantee if $n / d$ is large enough), we may fix a triangle-free graph $\hat{G}$ on $j$ vertices that has minimum degree at least $(2+\varepsilon / 4)^{-1 / 2} \sqrt{j \log j}$ and stability number at most $(2+\varepsilon / 4)^{1 / 2} \sqrt{j \log j}$. Form a new graph $G$ from $\hat{G}$ by replacing each vertex by a stable set of size $\lfloor n / j\rfloor$, and adding a complete bipartite graph between every pair of stable sets that corresponds to an edge in $\hat{G}$. Observe that $G$ is a triangle-free graph on between $n / 2$ and $n$ vertices with minimum degree at least $(2+\varepsilon / 4)^{-1 / 2} \sqrt{j \log j}\lfloor n / j\rfloor \geqslant d$. Moreover, $G$ has stability number at most $(2+\varepsilon / 4)^{1 / 2} \sqrt{j \log j}\lfloor n / j\rfloor \leqslant 2(2+\varepsilon / 4) d$ and so has fractional chromatic number at least $(n / 2) /(2(2+\varepsilon / 4) d)=n /((8+\varepsilon) d)$, as desired.

(Note that we essentially lost a factor 2 twice due to rounding, which is only an issue when $d=\Theta(\sqrt{n \log n})$. Thus when $d=\omega(\sqrt{n \log n})$ the $n / d$ upper bound is in fact correct up to a factor of $(2+o(1))$ as $n \rightarrow \infty$, and so in this case we can take the choice $C_{2}=1 / 2+o(1)$ in the theorem statement.)

For the other lower bound, recall that the triangle-free process on $n$ vertices yields, with high probability, a random graph with stability number at most $(\sqrt{2}+o(1)) \sqrt{n \log n}$. Thus the fractional chromatic number of that graph is at least $(2+o(1))^{-1 / 2} \sqrt{n / \log n}$.

\section{Bipartite induced density}

Here is a link between fractional colouring and bipartite induced density, and hence between Theorems 1.2 and 1.3.

Theorem 3.1 (Esperet, Kang, and Thomassé [10]). Any graph with fractional chromatic number at most $k$ and average degree $d$ has a bipartite induced subgraph of average degree at least $d / k$.

There is always a subgraph whose minimum degree is at least half the graph's average degree. Thus the lower bound in Theorem 1.2 follows from the upper bound in Theorem 1.3. 
Our next task, and the main task of this section, is to prove near sharpness in Theorem 1.3.

However, instead of a dense bipartite induced subgraph, one might be satisfied with a dense bipartite subgraph where we only require that (at least) one of the two parts induces a stable set. Given $G=(V, E)$, we call an induced subgraph $G^{\prime}=\left(V^{\prime}, E^{\prime}\right)$ of $G$ semi-bipartite if it admits a partition $V^{\prime}=V_{1} \cup V_{2}$ such that $V_{1}$ is a stable set of $G$, and we define the degree of a vertex of $G^{\prime}$ with respect to the semi-bipartition as its degree in the bipartite subgraph $G\left[V_{1}, V_{2}\right]$ between $V_{1}$ and $V_{2}$ (and so we ignore any edges in $V_{2}$ ). A version of Conjecture 1.1 where 'bipartite' is replaced by 'semi-bipartite' is known [10].

In what follows we give near optimal constructions for Theorem 1.2 not only for bipartite induced density, but also for semi-bipartite induced density. The following result, an adaptation of work of Spencer [23], is central. It might also be possible to adapt an earlier construction due to Erdős [7], but it would produce a construction comparable to Theorem 1.2. Although we chose not to pursue it, we suspect that the outcome at the end of the triangle-free process is significantly better, and optimal up to a constant factor. For this reason, we did not optimise either of the constants below.

Theorem 3.2. There exist constants $\delta, \gamma>0$ such that for every large enough $n$ there is a triangle-free graph on $n$ vertices with minimum degree at least $\delta \sqrt{n}$ that contains no semi-bipartite induced subgraph of minimum degree at least $\gamma \log n$.

Before proving this, let us see how it implies the second part of Theorem 1.2.

Proof of sharpness in Theorem 1.2. Let $j$ be the smaller of $n$ and the least value for which $2 \sqrt{j} \geqslant \delta n / d$. Provided $j$ is large enough, we may by Theorem 3.2 fix a triangle-free graph $\hat{G}$ that has minimum degree at least $\delta \sqrt{j}$ that contains no semi-bipartite induced subgraph of minimum degree at least $\gamma \log j$. Consider a new graph $G$ formed from $\hat{G}$ by replacing each vertex by a stable set of size $\lfloor n / j\rfloor$, and adding a complete bipartite graph between every pair of stable sets that corresponds to an edge in $\hat{G}$. Note that $G$ is a trianglefree graph on $j$ between $n / 2$ and $n$ vertices with minimum degree at least $\delta \sqrt{j}\lfloor n / j\rfloor \geqslant d$. Moreover, the largest minimum degree of a semi-bipartite induced subgraph in $G$ is smaller than $\gamma\lfloor n / j\rfloor \log j \leqslant \gamma\left\lceil\delta^{2} d^{2} /(16 n)\right\rceil \log n$.

For Theorem 3.2 we will need the convenient observation that, if we do not mind constant factors, it suffices to consider only semi-bipartite induced subgraphs with both parts of equal size.

Proposition 3.3 ([10]). Suppose $A, B \subseteq G$ are disjoint with $|A| \geqslant|B|$ and satisfy that the average degree of $G[A, B]$ is $d$. Then there exists $A^{\prime} \subseteq A$ with $\left|A^{\prime}\right|=|B|$ such that the average degree of $G\left[A^{\prime}, B\right]$ is at least $d / 2$.

Proof of Theorem 3.2. For a sufficiently large positive integer $n$, let $p=c_{1} / \sqrt{n}$ and $t=$ $c_{2} \sqrt{n} \log n$ for some fixed $c_{1}, c_{2}>0$. Consider the binomial random graph $G(n, p)$. Fix $0<\alpha<1$ and $\beta>0$. The constants $c_{1}, c_{2}, \alpha, \beta$ will be specified more precisely later in the proof.

With a view to applying the General Local Lemma (as stated in Subsection 1.1), let us define four types of (bad) events in $G(n, p)$. 
A For a set of three vertices, it induces a triangle.

B For a set of $t$ vertices, it induces a stable set.

C For a single vertex, it has degree at most $(1-\alpha) n p$.

$\mathrm{D}_{i}$ For two disjoint sets of $i$ vertices, the bipartite subgraph induced by the cut between the two sets has average degree at least $\beta \log n$.

Note that by Proposition 3.3, the fact that average degree is at least minimum degree, and the choice of $p$, we obtain the desired graph if there is an element of the probability space $G(n, p)$ for which no event of Types $\mathrm{A}, \mathrm{B}, \mathrm{C}$, and $\mathrm{D}_{i}, \beta \log n \leqslant i \leqslant t$, occur.

By an abuse of notation, let us write $P(*)$ for the probability of an event of Type $*$. To justify this abuse, observe that all events of Type $*$ have the same probability of occurrence. We have that $P(\mathrm{~A})=p^{3}$ and $P(\mathrm{~B})=(1-p)^{\left(\begin{array}{l}t \\ 2\end{array}\right)}<\exp \left(-p\left(\begin{array}{l}t \\ 2\end{array}\right)\right)$. Note that $P(\mathrm{C})=\mathbb{P}(\operatorname{Bin}(n, p) \leqslant(1-\alpha) n p) \leqslant 2 \exp \left(-\alpha^{2} n p / 3\right)$ by a Chernoff Bound (as stated in Subsection 1.1). Since $i p \leqslant t p \leqslant c_{1} c_{2} \log n, P\left(\mathrm{D}_{i}\right)=\mathbb{P}\left(\operatorname{Bin}\left(i^{2}, p\right) \geqslant \beta i \log n\right) \leqslant$ $\exp (-\beta i \log n)$ for all $i \leqslant t$ by a Chernoff Bound with a choice of $\beta$ satisfying

$$
\beta \geqslant 7 c_{1} c_{2}
$$

For Types $*$ and $*^{\prime}$, let us write $N\left(*, *^{\prime}\right)$ for the least such number for which each Type $*$ event is mutually independent of all but $N\left(*, *^{\prime}\right)$ events of Type $*^{\prime}$. We have that $N(\mathrm{~A}, \mathrm{~A})=3(n-3)<3 n, N(\mathrm{~B}, \mathrm{~A})=\left(\begin{array}{l}t \\ 2\end{array}\right)(n-t)+\left(\begin{array}{l}t \\ 3\end{array}\right)<t^{2} n / 2, N(\mathrm{C}, \mathrm{A})=\left(\begin{array}{c}n-1 \\ 2\end{array}\right)<n^{2} / 2$, and $N\left(\mathrm{D}_{i}, \mathrm{~A}\right)<i^{2} n$. More crudely, we can simply bound $N\left(*, *^{\prime}\right)$ by the number of Type $*^{\prime}$ events. This yields $N(\mathrm{~A}, \mathrm{~B}), N(\mathrm{~B}, \mathrm{~B}), N(\mathrm{C}, \mathrm{B})$, and $N\left(\mathrm{D}_{i}, \mathrm{~B}\right)$ are all at most $\left(\begin{array}{l}n \\ t\end{array}\right)<$ $(e n / t)^{t}=\exp (t \log (e n / t)) ; N(\mathrm{~A}, \mathrm{C}), N(\mathrm{~B}, \mathrm{C}), N(\mathrm{C}, \mathrm{C})$, and $N\left(\mathrm{D}_{i}, \mathrm{C}\right)$ are all at most $n$; and $N\left(\mathrm{~A}, \mathrm{D}_{j}\right), N\left(\mathrm{~B}, \mathrm{D}_{j}\right), N\left(\mathrm{C}, \mathrm{D}_{j}\right)$, and $N\left(\mathrm{D}_{i}, \mathrm{D}_{j}\right)$ are all at most $\left(\begin{array}{c}n \\ 2 j\end{array}\right)<(e n / 2 j)^{2 j}=$ $\exp (2 j \log (e n / 2 j))$,

By the General Local Lemma, we only need to find, for $\beta \log n \leqslant i \leqslant t$, positive reals $Y(\mathrm{~A}), Y(\mathrm{~B}), Y(\mathrm{C})$, and $Y\left(\mathrm{D}_{i}\right)$ such that the following inequalities hold:

$$
\begin{aligned}
1> & Y(\mathrm{~A}) P(\mathrm{~A}), Y(\mathrm{~B}) P(\mathrm{~B}), Y(\mathrm{C}) P(\mathrm{C}), Y\left(\mathrm{D}_{i}\right) P\left(\mathrm{D}_{i}\right) ; \\
\log Y(\mathrm{~A})> & Y(\mathrm{~A}) P(\mathrm{~A}) N(\mathrm{~A}, \mathrm{~A})+Y(\mathrm{~B}) P(\mathrm{~B}) N(\mathrm{~A}, \mathrm{~B})+ \\
& +Y(\mathrm{C}) P(\mathrm{C}) N(\mathrm{~A}, \mathrm{C})+\sum_{j} Y\left(\mathrm{D}_{j}\right) P\left(\mathrm{D}_{j}\right) N\left(\mathrm{~A}, \mathrm{D}_{j}\right) ; \\
\log Y(\mathrm{~B})> & Y(\mathrm{~A}) P(\mathrm{~A}) N(\mathrm{~B}, \mathrm{~A})+Y(\mathrm{~B}) P(\mathrm{~B}) N(\mathrm{~B}, \mathrm{~B})+ \\
& +Y(\mathrm{C}) P(\mathrm{C}) N(\mathrm{~B}, \mathrm{C})+\sum_{j} Y\left(\mathrm{D}_{j}\right) P\left(\mathrm{D}_{j}\right) N\left(\mathrm{~B}, \mathrm{D}_{j}\right) ; \\
\log Y(\mathrm{C})> & Y(\mathrm{~A}) P(\mathrm{~A}) N(\mathrm{C}, \mathrm{A})+Y(\mathrm{~B}) P(\mathrm{~B}) N(\mathrm{C}, \mathrm{B})+ \\
& +Y(\mathrm{C}) P(\mathrm{C}) N(\mathrm{C}, \mathrm{C})+\sum_{j} Y\left(\mathrm{D}_{j}\right) P\left(\mathrm{D}_{j}\right) N\left(\mathrm{C}, \mathrm{D}_{j}\right) ; \quad \text { and } \\
\log Y\left(\mathrm{D}_{i}\right)> & Y(\mathrm{~A}) P(\mathrm{~A}) N\left(\mathrm{D}_{i}, \mathrm{~A}\right)+Y(\mathrm{~B}) P(\mathrm{~B}) N\left(\mathrm{D}_{i}, \mathrm{~B}\right)+
\end{aligned}
$$




$$
+Y(\mathrm{C}) P(\mathrm{C}) N\left(\mathrm{D}_{i}, \mathrm{C}\right)+\sum_{j} Y\left(\mathrm{D}_{j}\right) P\left(\mathrm{D}_{j}\right) N\left(\mathrm{D}_{i}, \mathrm{D}_{j}\right)
$$

The estimates that we derived earlier imply that it instead suffices to find positive reals $Y(\mathrm{~A}), Y(\mathrm{~B}), Y(\mathrm{C})$, and $Y\left(\mathrm{D}_{i}\right)$ for which the following hold:

$$
\begin{aligned}
1 & >Y(\mathrm{~A}) p^{3}, \frac{Y(\mathrm{~B})}{\exp \left(p\left(\begin{array}{c}
t \\
2
\end{array}\right)\right)}, \frac{2 Y(\mathrm{C})}{\exp \left(\alpha^{2} n p / 3\right)}, \frac{Y\left(\mathrm{D}_{i}\right)}{\exp (\beta i \log n)} \\
Z & <\log Y(\mathrm{~A})-Y(\mathrm{~A}) p^{3} \cdot 3 n \\
Z & <\log Y(\mathrm{~B})-Y(\mathrm{~A}) p^{3} t^{2} n / 2 \\
Z & <\log Y(\mathrm{C})-Y(\mathrm{~A}) p^{3} n^{2} / 2 ; \text { and } \\
Z & <\log Y\left(\mathrm{D}_{i}\right)-Y(\mathrm{~A}) p^{3} i^{2} / n
\end{aligned}
$$

where

$$
\begin{array}{r}
Z=Y(\mathrm{~B}) \exp \left(-p\left(\begin{array}{l}
t \\
2
\end{array}\right)+t \log \frac{e n}{t}\right)+Y(\mathrm{C}) \cdot 2 \exp \left(-\alpha^{2} n p / 3\right) \cdot n+ \\
+\sum_{j} Y\left(\mathrm{D}_{j}\right) \exp (-\beta j \log n+2 j \log (e n / 2 j)) .
\end{array}
$$

Let us choose $Y(\mathrm{~A})=1+\varepsilon, Y(\mathrm{~B})=\exp \left(c_{3} \sqrt{n} \log ^{2} n\right), Y(\mathrm{C})=\exp \left(c_{4} \sqrt{n}\right)$, and $Y\left(\mathrm{D}_{i}\right)=$ $\exp \left(c_{5} i \log n\right)$ for some fixed $\varepsilon, c_{3}, c_{4}, c_{5}>0$. Note by the choices of $p$ and $t$ that the first row of inequalities are easily satisfied.

For the remaining rows, we first consider the asymptotic behaviour of the three constituents of $Z$ as $n \rightarrow \infty$. For the first two, we have

$$
\begin{aligned}
& Y(\mathrm{~B}) \exp \left(-p\left(\begin{array}{l}
t \\
2
\end{array}\right)+t \log \frac{e n}{t}\right)=\exp \left(\left(c_{3}-c_{1} c_{2}^{2} / 2+c_{2}+o(1)\right) \sqrt{n} \log ^{2} n\right) \\
& \text { and } Y(\mathrm{C}) \cdot 2 \exp \left(-\alpha^{2} n p / 3\right) \cdot n=\exp \left(\left(c_{4}-\alpha^{2} c_{1} / 3+o(1)\right) \sqrt{n}\right)
\end{aligned}
$$

For the third, note since $\beta \log n \leqslant j \leqslant t$ that

$$
\begin{aligned}
& \sum_{j} Y\left(\mathrm{D}_{j}\right) \exp (-\beta j \log n+2 j \log (e n / 2 j)) \\
& \leqslant \sum_{j} \exp \left(\left(c_{5}-\beta+2+o(1)\right) j \log n\right) \leqslant \exp \left(\left(c_{5}-\beta+2+o(1)\right) \beta \log ^{2} n\right) .
\end{aligned}
$$

We may therefore conclude that $Z$ is superpolynomially small in $n$ provided

$$
\begin{aligned}
c_{3}-c_{1} c_{2}^{2} / 2+c_{2} & <0, \\
c_{4}-\alpha^{2} c_{1} / 3 & <0, \text { and } \\
c_{5}-\beta+2 & <0 .
\end{aligned}
$$


Of the remaining terms in the inequalities required for the application of the General Local Lemma, the critical ones can be seen to be polylogarithmic or greater in magnitude $($ as $n \rightarrow \infty)$ :

$$
\begin{aligned}
& \log Y(\mathrm{~B})-Y(\mathrm{~A}) p^{3} t^{2} n / 2=\left(c_{3}-(1+\varepsilon) c_{1}^{3} c_{2}^{2} / 2+o(1)\right) \sqrt{n} \log ^{2} n \\
& \log Y(\mathrm{C})-Y(\mathrm{~A}) p^{3} n^{2} / 2=\left(c_{4}-(1+\varepsilon) c_{1}^{3} / 2+o(1)\right) \sqrt{n} ; \text { and } \\
& \log Y\left(\mathrm{D}_{i}\right)-Y(\mathrm{~A}) p^{3} i^{2} / n \geqslant\left(c_{5}-(1+\varepsilon) c_{1}^{3} c_{2}\right) i \log n
\end{aligned}
$$

(where we used $i \leqslant c_{2} \sqrt{n} \log n$ in the last line).

We therefore also want that

$$
\begin{aligned}
c_{3}-(1+\varepsilon) c_{1}^{3} c_{2}^{2} / 2 & >0 ; \\
c_{4}-(1+\varepsilon) c_{1}^{3} / 2 & >0 ; \text { and } \\
c_{5}-(1+\varepsilon) c_{1}^{3} c_{2} & >0 .
\end{aligned}
$$

It remains only to show that there is some choice of $c_{1}, \ldots, c_{5}, \alpha, \beta, \varepsilon$ so that (1)-(7) are fulfilled. Note that, whatever the other choices, the inequalities (1), (4), and (7) are satisfied with a sufficiently large choice of $\beta$ or of $c_{5}$. By pairing inequalities (2) and (5) as well as (3) and (6), we need

$$
\begin{aligned}
c_{2}\left(c_{1} c_{2} / 2-1\right) & >c_{3}>(1+\varepsilon) c_{1}^{3} c_{2}^{2} / 2 \text { and } \\
\alpha^{2} c_{1} / 3 & >c_{4}>(1+\varepsilon) c_{1}^{3} / 2 .
\end{aligned}
$$

We have that

$$
\begin{aligned}
c_{2}\left(c_{1} c_{2} / 2-1\right)>(1+\varepsilon) c_{1}^{3} c_{2}^{2} / 2 & \Longleftrightarrow c_{2}>2 /\left(c_{1}-(1+\varepsilon) c_{1}^{3}\right) \text { and } \\
\alpha^{2} c_{1} / 3>(1+\varepsilon) c_{1}^{3} / 2 & \Longleftrightarrow \alpha>\sqrt{3(1+\varepsilon) / 2} \cdot c_{1} .
\end{aligned}
$$

Let us then fix $c_{1}=1 / \sqrt{3}$. Therefore with a small enough choice of $\varepsilon>0$ it is possible to choose, say, $c_{2}=21 / 4$ and $\alpha=3 / 4$ ( $<1$ particular) and then take, say, $c_{3}=0.51$ and $c_{4}=0.97$. This completes the proof.

\section{Fractional colouring revisited}

In this section, we make a first step towards optimising the asymptotic constant for the first term in Theorem 1.3. It turns out that this is related to two problems of Erdös and Hajnal [8], concerning the asymptotic order of the chromatic number of a triangle-free graph with a given number of vertices or edges. In terms of edges, the correct order upper bound was first shown by Poljak and Tuza [21]. Matching lower bounds to settle both problems were established as byproduct to the determination of the asymptotic order of the Ramsey numbers $R(3, t)$ by Kim [17], cf. [12, 20].

For completeness we reiterate more precisely the observation of Erdös and Hajnal [8] mentioned in the proof of Theorem 1.3 (see also [14, pp. 124-5] and [17]). An application of Shearer's lower bound on the stability number [22] in a greedy colouring procedure bounds the chromatic number by at most $(4+o(1))$ times optimal (as certified by the triangle-free process $[3,11])$. 
Lemma 4.1 (cf. Jensen and Toft [14]). Let $\mathcal{G}$ be a class of graphs that is closed under vertex-deletion. Suppose that for some $x_{0} \geqslant 2$ there is a continuous, non-decreasing function $f_{\mathcal{G}}:\left[x_{0}, \infty\right) \rightarrow \mathbb{R}^{+}$such that every $G^{\prime} \in \mathcal{G}$ on $x \geqslant x_{0}$ vertices has a stable set of at least $f_{\mathcal{G}}(x)$ vertices. Then every $G \in \mathcal{G}$ on $n \geqslant x_{0}$ vertices has chromatic number at most

$$
x_{0}+\int_{x_{0}}^{n} \frac{d x}{f_{\mathcal{G}}(x)} .
$$

Corollary 4.2. As $n \rightarrow \infty$, any triangle-free graph on $n$ vertices has chromatic number at most $(2 \sqrt{2}+o(1)) \sqrt{n / \log n}$.

Proof. Shearer [22] showed that, for any $\varepsilon>0$, there exists $x_{0} \geqslant 2$ such that the function $f_{\mathcal{G}}(x)=(1 / \sqrt{2}-\varepsilon) \sqrt{x \log x}$ satisfies the hypothesis of Lemma 4.1 for $\mathcal{G}$ being the class of triangle-free graphs. Lemma 4.1 yields the desired outcome after an exercise in analysis to show that

$$
\lim _{n \rightarrow \infty} \frac{\int_{x_{0}}^{n} d x / \sqrt{x \log x}}{\sqrt{n / \log n}}=2 .
$$

Is the factor $(2+o(1))$ contribution from the above limit truly necessary? We were unable to address this issue, but Theorem 1.4 shows that it is possible to reduce the bound by a factor $(\sqrt{2}+o(1))$ if we only wish to bound the fractional chromatic number. Note that definitive progress on whether it is possible to improve by strictly more than a factor $(2+o(1))$ in Corollary 4.2, for fractional or not, either positively or negatively, would likely constitute a major breakthrough in combinatorics. A factor $(2+o(1))$ improvement is indeed plausible, especially for fractional.

Conjecture 4.3. As $n \rightarrow \infty$, any triangle-free graph on $n$ vertices has fractional chromatic number at most $(\sqrt{2}+o(1)) \sqrt{n / \log n}$.

Relatedly, in the spirit of [8], we also conjecture the following. Like for the previous conjecture, this if true would be an analogue of Shearer's bound.

Conjecture 4.4. As $m \rightarrow \infty$, any triangle-free graph with $m$ edges has fractional chromatic number at most $\left(2^{4 / 3}+o(1)\right) m^{1 / 3} /(\log m)^{2 / 3}$.

Here it is essential to mention a recent achievement of Molloy [19], a simpler derivation of and improvement upon Johansson's theorem [15].

Theorem 4.5 (Molloy [19]). As $\Delta \rightarrow \infty$, any triangle-free graph of maximum degree at most $\Delta$ has list chromatic number at most $(1+o(1)) \Delta / \log \Delta$.

Since this may be considered a stronger form of Shearer's bound, one might wonder if it alone is enough to verify Conjecture 4.3. This does not seem to be the case. We remark however that Theorems 1.3 and 4.5 together immediately yield Conjectures 4.3 and 4.4 for regular triangle-free graphs. (Let $G$ be a $D$-regular triangle-free graph. If $D \geqslant$ $\sqrt{2^{-1} n \log n}$, then it follows from Theorem 1.3; otherwise, it follows from Theorem 4.5.) 
Moreover, as we will shortly see, an iterated application of Theorem 4.5 combined with the simple idea in the proof of Theorem 1.3 yields Theorem 1.4.

Recall that we may equivalently define the fractional chromatic number of a graph as the smallest $k$ such that there is an assignment of measurable subsets of the interval $[0, k]$ (or rather of any subset of $\mathbb{R}$ of measure $k$ ) to the vertices such that each vertex is assigned a subset of measure 1 and subsets assigned to adjacent vertices are disjoint.

Proof of Theorem 1.4. Fix $\varepsilon>0$. Without loss of generality, assume $\varepsilon<1 / 2$. Let $G=(V, E)$ be a triangle-free graph on $n$ vertices and let $D \leqslant n$ be some positive integer to be specified later in the proof. We first associate $n$ disjoint intervals of measure $1 / D$ to each of the full neighbourhood sets (each of which is a stable set), and assign each such interval to its neighbourhood's vertices. By independently, arbitrarily de-assigning some (parts) of these intervals, we may assume each vertex of degree at least $D$ has an assignment of measure exactly 1 . On the other hand, the subgraph induced by vertices of measure less than 1 has maximum degree less than $D$. More precisely, let $V_{i}$ be the set of vertices of degree exactly $i$ in $G$, for $i<D$ : this initial partial fractional colouring gives each vertex of $V_{i}$ an assignment of measure exactly $i / D<1$. We have essentially shown how it suffices to restrict our attention in the remainder of the proof to $G$ having maximum degree $D$, by an incorporation of the same idea used in the proof of Theorem 1.3.

For each $D^{1 /(1+\varepsilon / 5)} \leqslant i<D$, let us write $G_{i}$ for the subgraph of $G$ induced by $\cup_{j=0}^{i} V_{j}$. Since $G_{i}$ is a triangle-free graph of maximum degree at most $i$, it follows from Theorem 4.5 that $G_{i}$ admits a proper colouring $c_{i}$ of its vertices with at most $(1+\varepsilon / 5) i / \log i$ colours, provided $i$ is large enough. (Since $i \geqslant D^{1 /(1+\varepsilon / 5)}, i$ is arbitrarily large if $D$ is.) For each colour class $\mathcal{C}$ of $c_{i}$, we choose an interval of measure $1 / D$ (that is disjoint from all previously used intervals), and assign it to each vertex of $\mathcal{C}$.

This extends the initial partial fractional colouring to nearly all of $G$. If $D^{1 /(1+\varepsilon / 5)} \leqslant$ $i<D$, then each vertex of $V_{i}$ has been assigned $D-i$ additional intervals of measure $1 / D$, resulting in an assignment of measure 1 . Note that, for $D$ large enough, the total measure of the subsets we have thus used is

$$
\begin{aligned}
\frac{n}{D} & +\frac{1}{D} \sum_{i=\left\lceil D^{1 /(1+\varepsilon / 5)}\right\rceil}^{D} \frac{(1+\varepsilon / 5) i}{\log i} \\
& \leqslant \frac{n}{D}+\frac{(1+\varepsilon / 5)^{2}}{D \log D} \sum_{i=0}^{D} i=\frac{n}{D}+\frac{(1+\varepsilon / 5)^{2}(D+1)}{2 \log D} \\
& \leqslant \frac{n}{D}+\frac{(1+\varepsilon / 2) D}{2 \log D} .
\end{aligned}
$$

We have extended the initial partial fractional colouring so that every vertex of $G$ has measure 1 apart from those vertices of degree less than $D^{1 /(1+\varepsilon / 5)}$. Since the above bound on the total measure used is strictly more than $D^{1 /(1+\varepsilon / 5)}$ if $D$ is large enough, we can greedily extend the partial fractional colouring to all remaining vertices without any additional measure. 
It remains to specify $D$ so that we use at most $(\sqrt{2}+\varepsilon) \sqrt{n / \log n}$ measure in total. Provided $n$ is large enough, the choice $D=\lfloor\sqrt{n \log n}\rfloor$ suffices. (Note that under this choice $D$ is arbitrarily large if $n$ is.)

To conclude the section, we comment that a straightforward substitution of Theorem 1.4 or Corollary 4.2 together with Theorem 4.5 into the proof by Gimbel and Thomassen [12] (the proofs in [20, 21] being slightly less efficient) yields the following bounds. The constants are roughly 2.5 and 3 times larger than the constant in Conjecture 4.4 .

Proposition 4.6. As $m \rightarrow \infty$, any triangle-free graph with $m$ edges has fractional chromatic number at most $\left(3^{5 / 3}+o(1)\right) m^{1 / 3} /(\log m)^{2 / 3}$ and has chromatic number at most $\left(3^{5 / 3} 2^{1 / 3}+o(1)\right) m^{1 / 3} /(\log m)^{2 / 3}$.

The approach for Theorem 1.4 could possibly be adapted to more directly improve upon Proposition 4.6, but we have not yet managed to do so.

\section{Bounds involving cubes}

In this section, we prove Theorem 1.5. We also make some additional observations that link our results with the fractional distance-3 chromatic number.

Let us first remark that, given the adjacency matrix $A$ of a graph $G$, the total number of directed three-edge walks in $G$ is the sum of all entries in the matrix $A^{3}$. The proof of Theorem 1.5 combines ideas from the proofs of Theorems 1.3 and of 3.1, without needing to bound the fractional chromatic number.

Proof of Theorem 1.5. Let $G=(V, E)$ be a triangle-free graph with $|V|=n$ and $|E|=m$ and suppose $G$ has $w_{3}$ directed three-edge walks. We write $q=w_{3} /(2 n m)$ and note that

$$
q=\frac{\sum_{x \in V} \sum_{v \in N(x)} \sum_{w \in N(v)} \operatorname{deg}(w)}{n \sum_{x \in V} \operatorname{deg}(x)} .
$$

Let $s_{1}, s_{2}$ be two vertices chosen uniformly at random and let $\mathbf{S}_{1}:=N\left(s_{1}\right)$ and $\mathbf{S}_{2}:=$ $N\left(s_{2}\right)$ denote their neighbourhoods, which are stable sets by triangle-freeness. Note that $\mathbb{E}\left(\left|\mathbf{S}_{1}\right|\right)=\mathbb{E}\left(\left|\mathbf{S}_{2}\right|\right)=\frac{1}{n} \sum_{x \in V} \operatorname{deg}(x)$, so also $\left.\frac{1}{2} \mathbb{E}\left(\left|\mathbf{S}_{1}\right|+\mid \mathbf{S}_{2}\right) \mid\right)=\frac{1}{n} \sum_{x \in V} \operatorname{deg}(x)$.

The number of edges in the subgraph induced by $\mathbf{S}_{1} \cup \mathbf{S}_{2}$ satisfies

$$
\begin{aligned}
& \mathbb{E}\left(\left|E\left(G\left[\mathbf{S}_{1} \cup \mathbf{S}_{2}\right]\right)\right|\right) \\
& =\sum_{S_{1}, S_{2} \subseteq[n]} \mathbb{P}\left(\left(\mathbf{S}_{1}=S_{1}\right) \cap\left(\mathbf{S}_{2}=S_{2}\right)\right) \cdot\left|E\left(G\left[S_{1} \cup S_{2}\right]\right)\right| \\
& =\sum_{S_{2}} \mathbb{P}\left(\mathbf{S}_{2}=S_{2}\right) \sum_{S_{1}} \mathbb{P}\left(\mathbf{S}_{1}=S_{1}\right) \cdot\left|E\left(G\left[S_{1} \cup S_{2}\right]\right)\right| \\
& =\sum_{S_{2}} \mathbb{P}\left(\mathbf{S}_{2}=S_{2}\right) \cdot \mathbb{E}\left(\left|E\left(G\left[\mathbf{S}_{1} \cup S_{2}\right]\right)\right|\right)=\frac{1}{n} \sum_{x \in V} \mathbb{E}\left(\left|E\left(G\left[\mathbf{S}_{1} \cup N(x)\right]\right)\right|\right)
\end{aligned}
$$




$$
\begin{aligned}
& =\frac{1}{n} \sum_{x \in V} \sum_{v \in N(x)} \mathbb{E}\left(\left|\mathbf{S}_{1} \cap N(v)\right|\right)=\frac{1}{n} \sum_{x \in V} \sum_{v \in N(x)} \sum_{w \in N(v)} \frac{\operatorname{deg}(w)}{n} \\
& =\frac{\sum_{x \in V} \sum_{v \in N(x)} \sum_{w \in N(v)} \operatorname{deg}(w)}{n \sum_{x \in V} \operatorname{deg}(x)} \cdot \frac{\mathbb{E}\left(\left|\mathbf{S}_{1}\right|+\left|\mathbf{S}_{2}\right|\right)}{2}=\frac{q}{2} \mathbb{E}\left(\left|\mathbf{S}_{1}\right|+\left|\mathbf{S}_{2}\right|\right) .
\end{aligned}
$$

By linearity of expectation,

$$
\mathbb{E}\left(\left|E\left(G\left[\mathbf{S}_{1} \cup \mathbf{S}_{2}\right]\right)\right|-\frac{q}{2}\left(\left|\mathbf{S}_{1}\right|+\left|\mathbf{S}_{2}\right|\right)\right) \geqslant 0 .
$$

It follows that there are two stable sets $S_{1}$ and $S_{2}$ of $G$ with at least $\frac{q}{2}\left(\left|S_{1}\right|+\left|S_{2}\right|\right)$ edges in the subgraph induced by $S_{1} \cup S_{2}$. Discarding the vertices of $S_{1} \cap S_{2}$ (if any exist) yields a bipartite induced subgraph of average degree at least $q$. Therefore $G$ contains a bipartite induced subgraph of minimum degree at least $q / 2$, as desired.

Next we indicate a mild improvement upon our bounds in terms of fractional distance3 colouring. Given a graph $G$, the cube $G^{3}$ of $G$ is the simple graph formed from $G$ by including all edges between vertices that are connected by a path in $G$ of length at most 3. The fractional distance-3 chromatic number of $G$ is the fractional chromatic number $\chi_{f}\left(G^{3}\right)$ of $G^{3}$. Observe that, if $G$ is triangle-free and $S$ is a stable set of $G^{3}$, then the union $\cup_{v \in S} N_{G}(v)$ of neighbourhood sets taken over $S$ is a stable set in $G$. In the proofs of Theorems 1.3 and 1.5, if we sample stable sets by taking such neighbourhood unions according to the distribution given by $\chi_{f}\left(G^{3}\right)$ rather than uniformly taking a neighbourhood set, then we obtain the following.

Theorem 5.1. We have that

- the upper bound in Theorem 1.3 holds with $\chi_{f}^{3} / d$ instead of $n / d$; and

- Theorem 1.5 holds with $w_{3} /\left(4 \chi_{f}^{3} m\right)$ or $d^{2} /\left(2 \chi_{f}^{3}\right)$ instead of $w_{3} /(4 n m)$;

where in each case $G$ denotes the corresponding triangle-free graph and $\chi_{f}^{3}=\chi_{f}\left(G^{3}\right)$ denotes its fractional distance-3 chromatic number.

Curiously, as the triangle-free process is sharp in Theorem 1.3, we obtain the following. (Perhaps this same result with distance-2 also holds.)

Corollary 5.2. With high probability, the final output of the triangle-free process has $\Omega(n)$ fractional distance-3 chromatic number as $n \rightarrow \infty$.

\section{List colouring}

In Section 4, we pursued sharper but fractional versions of the original problems of Erdös and Hajnal [8]. In another direction, the natural list colouring versions are open to the best of our knowledge. 
Conjecture 6.1. There are constants $C_{1}, C_{2}>0$ such that any triangle-free graph on $n$ vertices with $m$ edges has list chromatic number at most $C \sqrt{n / \log n}$ and at most $C_{2} m^{1 / 3} /(\log m)^{2 / 3}$.

Note that by a result of Alon [2], the two terms in Conjecture 6.1 are correct up to $\log n$ and $\log m$ factors, respectively.

Moreover, using a similar approach as for Theorem 1.4, here we give progress towards one of the statements in Conjecture 6.1, as embodied by Theorem 1.6. Theorem 1.6 is a combination of the bounds in Corollary 6.3 and Theorem 6.4 stated below.

We need a modest refinement of the aforementioned result of Alon.

Proposition 6.2. Any graph on $n \geqslant 2$ vertices with fractional chromatic number $\chi_{f}$ has list chromatic number at most $\left\lceil\chi_{f} \log n\right\rceil$.

Proof. This adapts a standard argument that we include for completeness. Let $G=(V, E)$ be a graph on $n \geqslant 2$ vertices with fractional chromatic number $\chi_{f}$. Let $k=\left\lceil\chi_{f} \log n\right\rceil$ and $L$ be a $k$-list-assignment of $G$. We write $L(V)=\bigcup_{u \in V} L(u)$ for the set of colours listed by $L$. By a standard equivalent definition of fractional colouring, there is a proper $(a, b)$ colouring $c$ of $G$ with $a / b=\chi_{f}$; that is, there is an assignment of subsets of $\{1, \ldots, a\}$ of size $b$ to the vertices of $G$ such that adjacent vertices are assigned disjoint subsets.

For each $x \in L(V)$ let $\mathbf{y}(x)$ be uniformly drawn from $\{1, \ldots, a\}$, and for each $u \in V$ let $\mathbf{L}_{c}(u)=L(u) \cap \bigcup_{i \in c(u)} \mathbf{y}^{-1}(i)$; that is, $\mathbf{L}_{c}(u)$ consists of those elements of $L(u)$ whose random choice from $\{1, \ldots, a\}$ is an element of the $b$-element set $c(u)$. Since necessarily $\mathbf{L}_{c}(u) \cap \mathbf{L}_{c}(v)=\emptyset$ if $u v \in E$, there is a proper $L$-colouring of $G$ if it holds that $\mathbf{L}_{c}(u) \neq \emptyset$ for all $u \in V$. But this follows from a union bound and the probabilistic method since for all $u$

$$
\mathbb{P}\left(\mathbf{L}_{c}(u)=\emptyset\right)=(1-b / a)^{k}<e^{-k / \chi_{f}} \leqslant 1 / n .
$$

Together with Theorem 1.3, this has the following direct consequence.

Corollary 6.3. Any triangle-free graph on $n$ vertices with minimum degree at least $d$ has list chromatic number at most $\lceil(n \log n) / d\rceil$.

Theorem 6.4. As $n \rightarrow \infty$, any triangle-free graph on $n$ vertices has list chromatic number at most $(2 \sqrt{2}+o(1)) \sqrt{n}$.

Proof. Fix $\varepsilon>0$. Without loss of generality, assume $\varepsilon<1 / 2$. Let $G=(V, E)$ be a triangle-free graph on $n$ vertices and choose $D=(1+o(1)) \sqrt{n / 2} \cdot \log n$ such that $(n \log n) / D=D / \log D=(1+o(1)) \sqrt{2 n}$. Just as in the proof of Theorem 1.4, we associate $n$ disjoint intervals of measure $1 / D$ to each of the full neighbourhood sets (each of which is a stable set), and assign each such interval to its neighbourhood's vertices. Each vertex of degree more than $D$ has an assignment of measure greater than 1 .

Writing $G_{\leqslant D}$ for the subgraph of $G$ induced by the vertices of degree at most $D$, and $G_{>D}=G-V\left(G_{\leqslant D}\right)$, we have just observed that $G_{>D}$ has fractional chromatic number less than $n / D$. By Proposition 6.2 and Theorem 1.3, it thus has list chromatic number at most $\lceil(n \log n) / D\rceil$. On the other hand, $G_{\leqslant D}$ has maximum degree $D$ and thus by 
Theorem 4.5 has list chromatic number at most $(1+\varepsilon / 4) D / \log D$ provided $D$ is large enough.

Let

$$
k=\left\lceil(1+\varepsilon)^{2}\left(\frac{n \log n}{D}+\frac{D}{\log D}\right)\right\rceil
$$

and $L$ be a $k$-list-assignment of $G$. We write $L(V)=\bigcup_{u \in V} L(u)$ for the set of colours listed by $L$. Next for each $x \in L(V)$ independently at random include $x$ in the list $\mathbf{L}_{\leqslant D}$ with probability $1 / 2$, and otherwise include $x$ in the list $\mathbf{L}_{>D}$. By a Chernoff Bound, for each $v \in V$,

$$
\begin{aligned}
\mathbb{P}\left(\left|L(v) \cap \mathbf{L}_{\leqslant D}\right|<\left(1+\frac{\varepsilon}{4}\right) \frac{D}{\log D}\right) & \leqslant \mathbb{P}\left(\left|L(v) \cap \mathbf{L}_{\leqslant D}\right| \leqslant(1-\varepsilon) \frac{k}{2}\right) \\
& \leqslant 2 \exp \left(-\varepsilon^{2} k / 6\right)=o(1 / n)
\end{aligned}
$$

as $n \rightarrow \infty$, using the choice of $D$ and the assumption that $\varepsilon<1 / 2$. Similarly,

$$
\mathbb{P}\left(\left|L(v) \cap \mathbf{L}_{>D}\right|<\left\lceil\frac{n \log n}{D}\right\rceil\right)=o(1 / n)
$$

A union bound and the probabilistic method guarantees for sufficiently large $n$ the existence of list-assignments $L_{\leqslant D}$ and $L_{>D}$ of $G$ such that the following properties hold for all $v \in V$ :

- $L(v)=L_{\leqslant D}(v) \cup L_{>D}(v)$ and $L_{\leqslant D}(v) \cap L_{>D}(v)=\emptyset$, and

- $\left|L_{\leqslant D}(v)\right| \geqslant(1+\varepsilon / 4) D / \log D$ and $\left|L_{>D}(v)\right| \geqslant\lceil(n \log n) / D\rceil$.

By the observations we made earlier, there is a proper $L_{\leqslant D}$-colouring of $G_{\leqslant D}$ and there is a proper $L_{>D}$-colouring of $G_{>D}$. Since these list-assignments are disjoint and are sublist-assignments of $L$, their combination constitutes a proper $L$-colouring of $G$. Since $k=\left((1+\varepsilon)^{2}+o(1)\right) 2 \sqrt{2 n}$, an arbitrarily small choice of $\varepsilon$ gives the result.

\section{Concluding remarks}

Although we were preoccupied with triangle-free graphs, one could naturally investigate graphs not containing $H$ as a subgraph for any fixed graph $H$. The following is in essence a more general form of Problem 4.1 in [10].

Problem 7.1. Given a graph $H$, is there $c_{H} \in(0,1)$ such that, as $n \rightarrow \infty$,

- if $c>c_{H}$, then any $H$-free graph on $n$ vertices with minimum degree $n^{c}$ has $n^{\Omega(1)}$ bipartite induced minimum degree; and

- if $c<c_{H}$, then there is an $H$-free graph on $n$ vertices with minimum degree $n^{c}$ and $O(\log n)$ bipartite induced minimum degree? 
We have shown that $c_{H}=1 / 2$ if $H$ is a triangle.

Problem 7.1 is particularly enticing when $H$ is the complete graph $K_{r}$ on $r \geqslant 4$ vertices. It was noted in [10] that the work of Ajtai, Komlós and Szemerédi [1] implies $c_{K_{r}} \leqslant 1-1 / r$ (if $c_{K_{r}}$ exists). It is possible to adapt Theorem 3.2 and [23] to show that $c_{K_{r}} \geqslant 1-(r-2) /\left(\left(\begin{array}{l}r \\ 2\end{array}\right)-1\right)$ (if $c_{K_{r}}$ exists). It is conceivable that $c_{K_{r}}=1-1 /(r-1)$. A motivation for this is that, even though prima facie there is no extremely close connection between bipartite induced density and large stable sets, we are tempted to speculate that, denoting the $H$ versus $K_{t}$ Ramsey number by $R\left(H, K_{t}\right)$,

$$
c_{H}=1-\lim _{t \rightarrow \infty} \frac{\log t}{\log R\left(H, K_{t}\right)} \text { (if } c_{H} \text { and the limit exist). }
$$

The righthand side is conjectured to be $1-1 /(r-1)$ when $H$ is $K_{r}$.

Motivated by Problem 7.1, we observe the following partial extensions of the bounds in Theorems 1.2 and 1.3.

Proposition 7.2. Fix an integer $r \geqslant 3$. For $r-2 \leqslant d \leqslant n / 2$, any $K_{1,1, r-2}$-free graph on $n$ vertices with minimum degree at least d has fractional chromatic number at most $\left(\begin{array}{c}n \\ r-2\end{array}\right) /\left(\begin{array}{c}d \\ r-2\end{array}\right)$, and thus contains a bipartite induced subgraph of minimum degree at least $\frac{d}{2}\left(\begin{array}{c}d \\ r-2\end{array}\right) /\left(\begin{array}{c}n \\ r-2\end{array}\right)$.

Proof. Let $G=(V, E)$ be a graph on $n$ vertices with minimum degree at least $d$ that contains no copy of $K_{1,1, r-2}$. We note that $K_{1,1, r-2}$-freeness implies that the joint neighbourhood of every vertex subset of size $r-2$ is a stable set. Choose $\mathbf{S}$ from the joint neighbourhood sets of $(r-2)$-vertex subsets uniformly over all $\left(\begin{array}{c}n \\ r-2\end{array}\right)$ such sets. Then it holds for all $v \in V$ that

$$
\left(\begin{array}{c}
n \\
r-2
\end{array}\right) \cdot \mathbb{P}(v \in \mathbf{S})=|\{T \subseteq N(v)|| T \mid=r-2\}| \geqslant\left(\begin{array}{c}
d \\
r-2
\end{array}\right) .
$$

We have shown then that this distribution has property $\mathrm{Q}_{\left(\begin{array}{c}d \\ r-2\end{array}\right) /\left(\begin{array}{c}n \\ r-2\end{array}\right)}^{*}$.

Note that the second part follows from Theorem 3.1.

Given a graph $H=\left(V_{H}, E_{H}\right)$ and a positive integer $x$, let us define

$$
\chi_{f}(H, x)=\max _{v \in V_{H}} \max \left\{\chi_{f}(J) \mid J \text { is an }(H-v) \text {-free graph on } x \text { vertices }\right\} .
$$

Proposition 7.3. Given a graph $H$, any $H$-free graph on $n$ vertices with minimum degree at least $d$ and maximum degree at most $\Delta$ has fractional chromatic number at most $\chi_{f}(H, \Delta) \cdot n / d$.

Proof. Let $G=(V, E)$ be a graph on $n$ vertices with minimum degree at least $d$ and maximum degree at most $\Delta$ that contains no copy of $H$. For any $v \in V$, there is by definition of $\chi_{f}(H, x)$ a distribution $\mathcal{S}_{v}$ over the stable sets of $N(v)$ such that any given $w \in N(v)$ is in $\mathbf{S}_{v}$ with probability at least $\left(\chi_{f}(H, \operatorname{deg}(v))\right)^{-1} \geqslant\left(\chi_{f}(H, \Delta)\right)^{-1}$ for a random $\mathbf{S}_{v}$ chosen according to $\mathcal{S}_{v}$. Let $\mathbf{S}$ be $\mathbf{S}_{v}$, where $v$ is uniformly chosen from $V$. Then any given $u \in V$ is in $\mathbf{S}$ with probability at least $\operatorname{deg}(u) /\left(n \cdot \chi_{f}(H, \Delta)\right)$, so this distribution has property $\mathrm{Q}_{d /\left(n \cdot \chi_{f}(H, \Delta)\right)}^{*}$. 
Let $P_{r}\left(C_{r}\right)$ denote a path (cycle, respectively) on $r \geqslant 2$ vertices. Every $P_{r}$-free graph is $(r-2)$-degenerate and therefore $(r-1)$-colourable. Thus $\chi_{f}\left(C_{r+1}, x\right) \leqslant r-1$ and we have the following corollary.

Corollary 7.4. Fix an integer $r \geqslant 3$. Any $C_{r}$-free graph on $n$ vertices with minimum degree $d$ has fractional chromatic number at most $(r-2) n / d$, and thus contains a bipartite induced subgraph of minimum degree at least $d^{2} /(2(r-2) n)$.

\section{Notes added}

Shortly after posting our manuscript to a public preprint repository, we learned that Matthew Kwan, Benny Sudakov and Tuan Tran independently obtained a finer version of Theorem 1.2 with different methods. In particular, they proved a bipartite induced minimum degree of order $\log d$ if $d=n^{\Omega(1)}$ and $d \leqslant \sqrt{n}$, and found a better construction in the case $d \geqslant n^{2 / 3}$. Later with Shoham Letzter [18] they moreover proved a marginally weaker form of Conjecture 1.1, guaranteeing a bipartite induced subgraph of minimum degree at least $C \log d / \log \log d$.

In a subsequent work, Kelly and Postle [16, Conj. 7.2] proposed a particular local strengthening of fractional colouring, in terms of local demands, and posed a conjecture in this context which would, if confirmed, establish Conjecture 4.3 as a corollary.

In a subsequent work of a subset of the present authors together with Davies [6], a generalisation of Conjecture 4.3 (from triangle-free graphs to graphs of a given local edge density) has been proposed.

The same subset of the present authors with Davies [5] have found a surprisingly short proof for the fractional colouring analogue of Theorem 4.5, via elementary properties of the hard-core model. Using this, there is a streamlined, self-contained proof (of about two pages overall) of Theorem 1.4.

\section{Acknowledgements}

We thank Ewan Davies for his insightful remark about regular graphs in Conjectures 4.3 and 4.4. We thank Matthew Kwan, Benny Sudakov and Tuan Tran for informing us of their independent, concurrent work and of the paper of Poljak and Tuza.

\section{References}

[1] M. Ajtai, J. Komlós, and E. Szemerédi. A dense infinite Sidon sequence. European J. Combin., 2(1):1-11, 1981.

[2] N. Alon. Choice numbers of graphs: a probabilistic approach. Combin. Probab. Comput., 1(2):107-114, 1992.

[3] T. Bohman and P. Keevash. Dynamic concentration of the triangle-free process. ArXiv e-prints, arXiv:1302.5963 Feb. 2013.

[4] S. Brandt and S. Thomassé. Dense triangle-free graphs are four-colorable: A solution to the Erdős-Simonovits problem. Manuscript, 2011. 
[5] E. Davies, R. de Joannis de Verclos, R. J. Kang, and F. Pirot. Colouring triangle-free graphs with local list sizes. ArXiv e-prints, arXiv:1812.01534 Dec. 2018. To appear in Random Structures \& Algorithms.

[6] E. Davies, R. de Joannis de Verclos, R. J. Kang, and F. Pirot. Occupancy fraction, fractional colouring, and triangle fraction. ArXiv e-prints, arXiv:1812.11152 Dec. 2018.

[7] P. Erdős. Graph theory and probability. II. Canad. J. Math., 13:346-352, 1961.

[8] P. Erdös and A. Hajnal. Chromatic number of finite and infinite graphs and hypergraphs. Discrete Math., 53:281-285, 1985. Special volume on ordered sets and their applications (L'Arbresle, 1982).

[9] P. Erdős and M. Simonovits. On a valence problem in extremal graph theory. Discrete Math., 5:323-334, 1973.

[10] L. Esperet, R. J. Kang, and S. Thomassé. Separation Choosability and Dense Bipartite Induced Subgraphs. Combin. Probab. Comput., 28(5):720-732, 2019.

[11] G. Fiz Pontiveros, S. Griffiths, and R. Morris. The Triangle-Free Process and the Ramsey Number $R(3, k)$. Memoirs of the American Mathematical Society, 263(1274), Jan. 2020.

[12] J. Gimbel and C. Thomassen. Coloring triangle-free graphs with fixed size. Discrete Math., 219(1-3):275-277, 2000.

[13] S. Janson, T. Łuczak, and A. Rucinski. Random Graphs. Wiley-Interscience Series in Discrete Mathematics and Optimization. Wiley-Interscience, New York, 2000.

[14] T. R. Jensen and B. Toft. Graph coloring problems. Wiley-Interscience Series in Discrete Mathematics and Optimization. John Wiley \& Sons, Inc., New York, 1995. A Wiley-Interscience Publication.

[15] A. Johansson. Asymptotic choice number for triangle-free graphs. Technical Report 91-5, DIMACS, 1996.

[16] T. Kelly and L. Postle. Fractional coloring with local demands. ArXiv e-prints, arXiv:1811.11806 2018.

[17] J. H. Kim. The Ramsey number $R(3, t)$ has order of magnitude $t^{2} / \log t$. Random Structures Algorithms, 7(3):173-207, 1995.

[18] M. Kwan, S. Letzter, B. Sudakov, and T. Tran. Dense Induced Bipartite Subgraphs in Triangle-Free Graphs. Combinatorica, 40(2):283-305, 2020.

[19] M. Molloy. The list chromatic number of graphs with small clique number. J. Combin. Theory Ser. B, 134:264-284, 2019.

[20] A. Nilli. Triangle-free graphs with large chromatic numbers. Discrete Math., 211(13):261-262, 2000.

[21] S. Poljak and Z. Tuza. Bipartite subgraphs of triangle-free graphs. SIAM J. Discrete Math., 7(2):307-313, 1994. 
[22] J. B. Shearer. A note on the independence number of triangle-free graphs. Discrete Math., 46(1):83-87, 1983.

[23] J. Spencer. Asymptotic lower bounds for Ramsey functions. Discrete Math., 20(1):6976, 1977/78.

[24] C. Thomassen. On the chromatic number of triangle-free graphs of large minimum degree. Combinatorica, 22(4):591-596, 2002. 